\title{
ON THE DECOUPLING AND OUTPUT FUNCTIONAL CONTROLLABILITY OF ROBOTIC MANIPULATION
}

\author{
D. Prattichizzo, ${ }^{*}$ P. Mercorelli, ${ }^{* *}$ A. Bicchi,${ }^{*}$ A. Vicino ${ }^{* *}$ \\ * Dipartimento di Sistemi Elettrici ed Automazione, Università di Pisa \\ via Diotisalvi, 2, 56100 Pisa, Italia \\ prattichizzo(bicchi)@dsea.unipi.it \\ ** Dipartimento di Ingegneria dell'Informazione, Università di Siena \\ via Roma, 56, 53100 Siena, Italia \\ mercorelli(vicino)@unisi.it \\ IFAC Symposium on Robot Control '97, SYROCO'97 \\ Nantes, France. September 1997
}

\begin{abstract}
Résumé : The problem of force/motion control in robotic manipulation is studied. A noninteracting control law of grasping contact forces and object motions is proposed. In order to achieve force/motion trajectory tracking without transient errors, the output functional controllability of the controlled output is investigated. The framework throughout is the geometric approach to the multivariable systems. Numerical examples are provided to show the effectiveness of the proposed control law.
\end{abstract}

Mont Clefs : Manipulators, force/motion control, decoupling, functional controllability.

\section{INTRODUCTION}

The class of robotic systems this paper is focused on is enough general to include mechanical structures more complex than conventional serial-linkage arms. Robotic hands can be considered as paradigms of this class.

The presence of unilateral contact phenomena between different parts of the mechanical structure is a special feature of manipulation systems. Mechanical contacts between the robotic parts and the environment can be viewed as unactuated (passive) joints and make manipulation system control quite involved.

The analysis of dynamics and the control of manipulation systems becomes more complex when it is not possible to control contact forces in all directions. This usually happens when the number of DoF's of the robotic device is smaller than the dimension of the contact force space. In (Prattichizzo and Bicchi, 1997; Prattichizzo and Bicchi, 1996), such a case is defined as "defective grasp". In industrial applications, kinematic defectivity is a common factor of almost all grippers used to grasp industrial parts, see Fig. 2.

The main goal of dexterous manipulation tasks consists of controlling the motion of the manipulated object along with the grasping forces exerted on the object. In the robotics literature, the general problem of force/motion control is known as "hybrid control". For a broad overview on this topics, the reader is referred to (Siciliano, 1996) and the references therein.

The decoupling property is a necessary requirement of the force/motion control law for all those advanced tasks, including micro manipulation of tissues in surgery and in laparoscopy or assembly and manipulation of non-rigid (rubber or plastic) parts in industry, where it could be very dangerous to change the squeezing force while giving rise to undesired transient object motions. 
In (Prattichizzo et al., 1996), the authors proved in a geometric setting (Basile and Marro, 1992) that it is possible to decouple the object position and the squeezing force control for a wide class of manipulation systems by using a state-space feedback controller. Here, the decoupling problem is investigated thoroughly in order to extend previous results to the functional force/motion controllability. The relevance of output functional controllability to manipulation control is justified by the necessity of very fast control loops to counteract grasp failures caused by possible external disturbances.

\section{PRELIMINARIES}

The manipulation system dynamics is linearized at an equilibrium configuration. For a detailed discussion of dynamics and the derivation of its linearized model the reader is referred to previous works by the authors, (Prattichizzo and Bicchi, 1997) and (Prattichizzo and Bicchi, 1996).

Notation and some results on the linearized dynamics of general manipulation systems, are summarized in this section. Let $\mathbf{q} \in \mathbb{R}^{q}$ be the vector of joint positions, $\tau \in \mathbb{R}^{q}$ the vector of joint forces and/or torques, $\mathbf{u} \in \mathbb{R}^{d}$ the vector locally describing the position and the orientation of a frame attached to the object and $\mathbf{w} \in \mathbb{R}^{d}$ the vector of external disturbances acting on the object. Finally let us introduce the vector $\mathbf{t} \in \mathbb{R}^{t}$ including forces and torques at all contacts.

Assume that contact forces arise from a lumpedparameter model of visco-elastic phenomena at the contacts, summarized by the stiffness matrix $\mathbf{K}$ and the damping matrix $\mathbf{B}$. The Jacobian $\mathbf{J}$ and the grasp matrix $\mathbf{G}$ are defined as usual as the linear maps relating the velocities of the contact points on the links and on the object, to the joint and object velocities, respectively.

The visco-elastic contact model is mandatory in all those tasks where contact stiffness is not negligible or in hyperstatic grasps (Prattichizzo and Bicchi, 1997) where the rigid-body contact model leaves the dynamics undetermined.

Consider a reference equilibrium configuration $(\mathbf{q}, \mathbf{u}, \dot{\mathbf{q}}, \dot{\mathbf{u}}, \tau, \mathbf{t})=\left(\mathbf{q}_{o}, \mathbf{u}_{o}, \mathbf{0}, \mathbf{0}, \tau_{o}, \mathbf{t}_{o}\right)$, such that $\tau_{o}=\mathbf{J}^{T} \mathbf{t}_{o}$ and $\mathbf{w}_{o}=-\mathbf{G t}_{o}$. In the neighbourhood of such an equilibrium the linearized dynamics of the manipulation system can be written as

$$
\dot{\mathbf{x}}=\mathbf{A x}+\mathbf{B}_{\tau} \tau^{\prime}+\mathbf{B}_{w} \mathbf{w}^{\prime},
$$

where state, input and disturbance vectors are defined as the departures from the reference equilibrium configuration:

$$
\begin{gathered}
\mathbf{x}=\left[\left(\mathbf{q}-\mathbf{q}_{o}\right)^{T}\left(\mathbf{u}-\mathbf{u}_{o}\right)^{T} \dot{\mathbf{q}}^{T} \dot{\mathbf{u}}^{T}\right]^{T} \\
\tau^{\prime}=\tau-\mathbf{J}^{T} \mathbf{t}_{o}, \mathbf{w}^{\prime}=\mathbf{w}+\mathbf{G} \mathbf{t}_{o} \text { and } \\
\mathbf{A}=\left[\begin{array}{cc}
\mathbf{0} & \mathbf{I} \\
\mathbf{L}_{k} & \mathbf{L}_{b}
\end{array}\right] ; \mathbf{B}_{\tau}=\left[\begin{array}{c}
\mathbf{0} \\
\mathbf{0} \\
\mathbf{M}_{h}^{-1} \\
\mathbf{0}
\end{array}\right] ; \mathbf{B}_{w}=\left[\begin{array}{c}
\mathbf{0} \\
\mathbf{0} \\
\mathbf{0} \\
\mathbf{M}_{o}^{-1}
\end{array}\right]
\end{gathered}
$$

being $\mathbf{M}_{h}$ and $\mathbf{M}_{o}$ the inertia matrices of the manipulator and the object, respectively. To simplify notation the prime in $\tau^{\prime}$ and $\mathbf{w}^{\prime}$ will be omitted.

Neglecting rolling phenomena at the contacts and assuming that local variations of the Jacobian, grasp and visco-elastic matrices are small, simple expressions are obtained for $\mathbf{L}_{k}=-\mathbf{M}^{-1} \mathbf{P}_{k}$ and $\mathbf{L}_{b}=-\mathbf{M}^{-1} \mathbf{P}_{b}$, where $\mathbf{M}=\operatorname{diag}\left(\mathbf{M}_{h}, \mathbf{M}_{o}\right)$, $\mathbf{P}_{k}=\mathbf{S}^{T} \mathbf{K S}, \mathbf{P}_{b}=\mathbf{S}^{T} \mathbf{B S}$, and $\mathbf{S}=\left[\mathbf{J}-\mathbf{G}^{T}\right]$.

\section{CONTROLLED OUTPUTS AND NONINTERACTING CONTROL}

In this paper, it has been assumed that contact points do not change. The manipulation is studied in those interval of time when contact points hold without rolling and/or sliding.

With reference to the first control output let us introduce the internal forces. They are self-balanced forces, belong to the null space of the grasp matrix $\mathbf{G}$ and enable the robotic device to grasp the object.

With reference to object trajectories, rigid-body kinematics play a particular function in manipulation control. Rigid-body kinematics have been studied in a quasi-static setting in (Bicchi et al., 1995) and in terms of unobservable subspaces in (Bicchi and Prattichizzo, 1995). In both cases rigid kinematics were described by the base matrix $\boldsymbol{\Gamma}$ whose columns form a basis for $\operatorname{ker}\left[\mathbf{J}-\mathbf{G}^{T}\right]=\operatorname{im}(\boldsymbol{\Gamma})$ where

$$
\boldsymbol{\Gamma}=\left[\begin{array}{ll}
\boldsymbol{\Gamma}_{q c}^{T} & \boldsymbol{\Gamma}_{u c}^{T}
\end{array}\right]^{T}, \quad \text { and } \quad \mathbf{J} \boldsymbol{\Gamma}_{q c}=\mathbf{G}^{T} \boldsymbol{\Gamma}_{u c} .
$$

Observe that, for the sake of brevity, it is assumed here that the system is not redundant: $\operatorname{ker}(\mathbf{J})=\{\mathbf{0}\}$ and that it is not indeterminate: $\operatorname{ker}\left(\mathbf{G}^{T}\right)=\{\mathbf{0}\}$, see (Bicchi et al., 1995) for further details.

The column space of $\boldsymbol{\Gamma}$ consists of coordinated rigid-body motions of the mechanism, for the manipulator $\left(\boldsymbol{\Gamma}_{q c}\right)$ and the object $\left(\boldsymbol{\Gamma}_{u c}\right)$ components. They do not involve visco-elastic deformations at contacts and can be regarded as low-energy motions.

In the following, a special subspace of internal forces and the rigid-body object motions are characterized as output matrices of the linearized dynamics, see Section 2. These outputs, namely $\mathbf{t}^{\prime}$ and $\mathbf{u}^{\prime}$ (henceforth $\mathbf{t}$ and $\mathbf{u}$ ), represent variations 


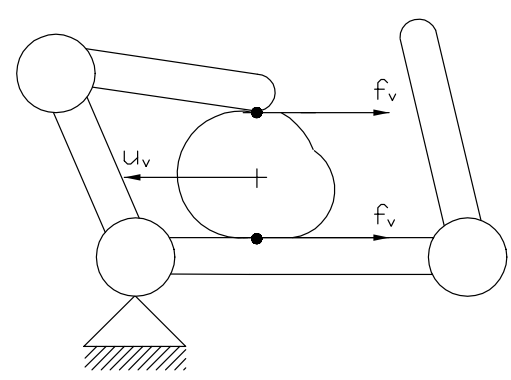

Fig. 1. Defective grasp: $\operatorname{ker}\left(\mathbf{J}^{T}\right) \neq\{0\}$. Contact force $\mathbf{f}_{v}$ and object position $\mathbf{u}_{v}$ which are not controllable by joint torques.

of contact force and object position vectors from the relative equilibrium values.

Before introducing the controlled outputs, let us recall the concept of contact-kinematics defectivity, or briefly defectivity. According to (Prattichizzo and Bicchi, 1996) and (Prattichizzo et al., 1996), a given grasp is called contact-kinematics defective if $\operatorname{ker}\left(\mathbf{J}^{T}\right) \neq\{\mathbf{0}\}$. Contact force and object motion controllability is generally lost in the presence of defectivity. Fig. 1 pictorially describes some uncontrollable directions of contact forces $\mathbf{f}_{v}$ and object motions $\mathbf{u}_{v}$ for a simple 3-DoF's defective device.

Although, in the presence of defectivity, contact forces $\mathbf{t}$ and object motions $\mathbf{u}$ loose the output controllability, it was shown in (Prattichizzo and Bicchi, 1996) that the output controllability property holds for their projection on the subspace of reachable internal forces $\mathbf{t}_{i}$ and of rigid-body object motions $\mathbf{u}_{c}$. Moreover, if the output vector is chosen by grouping such projections: $\mathbf{y}=\left(\mathbf{t}_{i}^{T} \mathbf{u}_{c}^{T}\right)^{T}$, the system result square and output controllable.

The reachable internal contact forces $\mathbf{t}_{i}$ are defined as the projection of the force vector $\mathbf{t}$ onto the null space of $\mathbf{G}$ :

$$
\begin{aligned}
& \mathbf{t}_{i}=\mathbf{E}_{t i} \mathbf{x} ; \quad \text { where } \\
& \mathbf{E}_{t i}=\left(\mathbf{Q}^{T} \mathbf{Q}\right)^{-1} \mathbf{Q}^{T}\left[\begin{array}{llll}
\mathbf{Q} & \mathbf{0} & \mathbf{Q} & \mathbf{0}
\end{array}\right] \text { and } \\
& \mathbf{Q}=\left(\mathbf{I}-\mathbf{K G}^{T}\left(\mathbf{G K G}^{T}\right)^{-1} \mathbf{G}\right) \mathbf{K} \mathbf{J}
\end{aligned}
$$

and the rigid-body object motions $\mathbf{u}_{c}$ are defined as the projection of the object displacement $\mathbf{u}$ onto the column space of $\Gamma_{u c}$ :

$$
\begin{aligned}
& \mathbf{u}_{c}=\mathbf{E}_{u c} \mathbf{X} ; \quad \text { where } \\
& \mathbf{E}_{u c}=\left(\Gamma_{u c}^{T} \Gamma\right)^{-1} \boldsymbol{\Gamma}_{u c}^{T}\left[\begin{array}{llll}
\mathbf{0} & \mathbf{1} & \mathbf{0} & \mathbf{0}
\end{array}\right] .
\end{aligned}
$$

To simplify notation, matrices $\left(\mathbf{Q}^{T} \mathbf{Q}\right)^{-1}$ and $\left(\Gamma_{u c}^{T} \Gamma\right),{ }^{-1}$ will be omitted.

\subsection{Noninteracting control}

In (Prattichizzo et al., 1996) the following decoupling theorem was stated.

Theorem 1. (Noninteraction) Consider the linearized manipulation system of Section 2. If $\operatorname{ker}\left(\mathbf{G}^{T}\right)=\{\mathbf{0}\}$, there exists a stabilizing statefeedback control law, $\tau=\mathbf{F} \mathbf{x}+\tau^{*}$ and an input partition $\tau^{*}=\mathbf{U}_{t i} \mathbf{u}_{t i}+\mathbf{U}_{u c} \mathbf{u}_{u c}$ (see Fig. 3) which decouples reachable internal forces $\mathbf{t}_{i}$ and rigidbody object motions $\mathbf{u}_{c}$.

Theorem 1 states that a control law and a joint torques partition exists such that, for zero initial conditions, each input only affects the relative output. The geometric concept from which the previous result develops is the $\mathcal{S}$-constrained controllability. It consists of those state space vectors reachable through trajectories entirely lying in the constraining subspace $\mathcal{S}$.

It was shown that, for the aforementioned outputs $\mathbf{t}_{i}$ and $\mathbf{u}_{c}$, there exists a decoupling and stabilizing state feedback matrix $\mathbf{F}$, along with two input partition matrices $\mathbf{U}_{t i}$ and $\mathbf{U}_{u c}$ such that, for the dynamic triples

$$
\begin{aligned}
& \left(\mathbf{E}_{t i}, \mathbf{A}+\mathbf{B}_{\tau} \mathbf{F}, \mathbf{B}_{\tau} \mathbf{U}_{t i}\right) ; \\
& \left(\mathbf{E}_{u c}, \mathbf{A}+\mathbf{B}_{\tau} \mathbf{F}, \mathbf{B}_{\tau} \mathbf{U}_{u c}\right)
\end{aligned}
$$

it holds:

$$
\begin{aligned}
& \mathcal{R}_{t i}=\min \mathcal{I}\left(\mathbf{A}+\mathbf{B}_{\tau} \mathbf{F}, \mathbf{B}_{\tau} \mathbf{U}_{t i}\right) \subseteq \operatorname{ker}\left(\mathbf{E}_{u c}\right) \\
& \mathbf{E}_{t i} \mathcal{R}_{t i}=\operatorname{im}\left(\mathbf{E}_{t i}\right) \\
& \mathcal{R}_{u c}=\min \mathcal{I}\left(\mathbf{A}+\mathbf{B}_{\tau} \mathbf{F}, \mathbf{B}_{\tau} \mathbf{U}_{u c}\right) \subseteq \operatorname{ker}\left(\mathbf{E}_{t i}\right) \\
& \mathbf{E}_{u c} \mathcal{R}_{u c}=\operatorname{im}\left(\mathbf{E}_{u c}\right) .
\end{aligned}
$$

Here, $\min \mathcal{I}(\mathbf{A}, \operatorname{im}(\mathbf{B}))=\sum_{i=0}^{n-1} \mathbf{A}^{i} \operatorname{im}(\mathbf{B})$ is the minimum $\mathbf{A}$-invariant subspace containing im $(\mathbf{B})$ and $\max \mathcal{I}(\mathbf{A}, \operatorname{ker}(\mathbf{C}))=\bigcap_{i=0}^{n-1} \mathbf{A}^{i} \operatorname{ker}(\mathbf{C})$ is the maximum $\mathbf{A}$-invariant subspace contained in $\operatorname{ker}(\mathbf{C})$ with respect to the triple $(\mathbf{A}, \mathbf{B}, \mathbf{C})$.

Moreover, partition matrices $\mathbf{U}_{u c}$ and $\mathbf{U}_{t i}$ satisfy the following relationships

$$
\begin{aligned}
& \operatorname{im}\left(\mathbf{B}_{\tau} \mathbf{U}_{u c}\right)=\operatorname{im}\left(\mathbf{B}_{\tau}\right) \cap \mathcal{R}_{u c} ; \\
& \operatorname{im}\left(\mathbf{B}_{\tau} \mathbf{U}_{t i}\right)=\operatorname{im}\left(\mathbf{B}_{\tau}\right) \cap \mathcal{R}_{t i}
\end{aligned}
$$

and the stabilizing matrix $\mathbf{F}$ is such that

$$
\begin{aligned}
& \left(\mathbf{A}+\mathbf{B}_{\tau} \mathbf{F}\right) \mathcal{R}_{u c} \subseteq \mathcal{R}_{u c} \\
& \left(\mathbf{A}+\mathbf{B}_{\tau} \mathbf{F}\right) \mathcal{R}_{t i} \subseteq \mathcal{R}_{t i} .
\end{aligned}
$$

In (Prattichizzo et al., 1997), under the assumption that

$\mathbf{A 1}: \operatorname{ker}\left(\boldsymbol{\Gamma}_{u c}^{T}\right)$ is $\mathbf{M}_{o}^{-1} \mathbf{G K G}^{T}$-invariant, the subspaces of constrained-reachability are obtained as 


$$
\begin{aligned}
& \mathcal{R}_{t i}=\operatorname{im}\left(\mathbf{R}_{t i}\right), \\
& \mathbf{R}_{t i}=\left[\begin{array}{cccccc}
\boldsymbol{\Gamma}_{h} & \mathbf{0} & \mathbf{X} & \mathbf{0} & \mathbf{0} & \mathbf{0} \\
\mathbf{0} & \mathbf{0} & \mathbf{0} & \mathbf{0} & \mathbf{P} & \mathbf{0} \\
\mathbf{0} & \boldsymbol{\Gamma}_{h} & \mathbf{0} & \mathbf{X} & \mathbf{0} & \mathbf{0} \\
\mathbf{0} & \mathbf{0} & \mathbf{M}_{o}^{-1} \mathbf{G K J X} & \mathbf{0} & \mathbf{0} & \mathbf{P}
\end{array}\right] \\
& \mathcal{R}_{u c}=\operatorname{im}\left(\mathbf{R}_{u c}\right), \\
& \mathbf{R}_{u c}=\left[\begin{array}{cccc}
\boldsymbol{\Gamma}_{q c} & \mathbf{0} & \mathbf{0} & \mathbf{0} \\
\mathbf{0} & \mathbf{0} & \mathbf{D} & \mathbf{0} \\
\mathbf{0} & \boldsymbol{\Gamma}_{q c} & \mathbf{0} & \mathbf{0} \\
\mathbf{0} & \mathbf{0} & \mathbf{0} & \mathbf{D}
\end{array}\right]
\end{aligned}
$$

where $\boldsymbol{\Gamma}_{h}, \mathbf{Y}, \mathbf{X}, \mathbf{P}$ and $\mathbf{D}$ are defined as follows

$$
\begin{aligned}
& \boldsymbol{\Gamma}_{h}=\mathbf{M}_{h}^{-1} \mathbf{J}^{T} \cap \max \mathcal{I}\left(\mathbf{M}_{h}^{-1} \mathbf{J}^{T} \mathbf{K J}, \operatorname{ker}(\mathbf{G K J})\right) ; \\
& \mathbf{Y}=\max \mathcal{I}\left(\mathbf{M}_{o}^{-1} \mathbf{G K G}^{T}, \operatorname{ker}\left(\boldsymbol{\Gamma}_{u c}^{T}\right)\right) \text {; } \\
& \mathbf{X} \text { s.t. } \mathbf{M}_{o}^{-1} \mathbf{G K J \mathbf { X }}=\mathbf{Y} \cap \mathbf{M}_{o}^{-1} \mathbf{G K J} \text {; } \\
& \mathbf{P}=\min \mathcal{I}\left(\mathbf{M}_{o}^{-1} \mathbf{G} \mathbf{K G}^{T}, \mathbf{M}_{o}^{-1} \mathbf{G K J X}\right) ; \\
& \mathbf{D}=\min \mathcal{I}\left(\mathbf{M}_{o}^{-1} \mathbf{G K G}{ }^{T}, \mathbf{M}_{o}^{-1} \mathbf{G K J} \boldsymbol{\Gamma}_{q c}\right) \text {. }
\end{aligned}
$$

where notation is abused to indicate matrices and corresponding column spaces as well.

\section{FORCE/MOTION FUNCTIONAL CONTROLLABILITY}

This section is aimed at the control of internal contact forces and rigid-body object motions to achieve tracking without transients of force and motion error variables. It is our belief that noninteraction should be a basic requirement of internalforce and object-motion control, thus the objective of the control becomes twofold and an effort is made to achieve both decoupling and functional controllability of reachable internal forces and rigid body motions in manipulation systems.

To attack the problem, the natural approach is to analyze the constrained output controllability idea, cf. (Basile and Marro, 1971) and (Basile and Marro, 1992), formalized below.

\section{Definition 1. (Perfect output controllability)} Given the triple $(\mathbf{A}, \mathbf{B}, \mathbf{C})$, the output subspace $\mathcal{L}^{i}$ is said to be perfect output functionally $(\mathrm{OF})$ controllable with respect to $i$-th derivative and with respect to the subspace of states $\mathcal{S}$ if $\mathcal{L}^{i}=\mathbf{C S}$ and, for every initial state $\mathbf{x}_{0} \in \mathcal{S}$, it is possible, by means of proper bounded and measurable control function, to follow in $\mathcal{L}^{i}$ any trajectory arbitrarily given in the class of functions which admit i-th derivative with respect to time, while the state evolves into $\mathcal{S}$.

Next theorem, whose proof is in Appendix, states the $\mathrm{OF}$-controllability of general manipulation systems decoupled according to the previous section.
Theorem 2. (Output Functional Controllability and Noninteraction) Consider the linearized System (1) with $\operatorname{ker}\left(\mathbf{G}^{T}\right)=\{\mathbf{0}\}$. Under assumption $\mathbf{A 1}$, the output subspaces $\operatorname{im}\left(\mathbf{E}_{t i}\right)$ and $\operatorname{im}\left(\mathbf{E}_{u c}\right)$ are $\mathrm{OF}$ controllable with respect to the 1st and 3 rd derivative and with respect to the constrained reachable subspaces $\mathcal{R}_{t i}$ and $\mathcal{R}_{u c}$, respectively. Moreover the state-feedback decoupling controller of Section 3.1 (eq. 5, 8 and 9) makes the system, with outputs $\mathbf{t}_{i}$ and $\mathbf{u}_{c}$, noninteracting and $\mathrm{OF}-$ controllable.

Remark 1. Regarding the functional controllability of rigid-body object motions, the 3 -rd order of derivative means that the output $\mathbf{u}_{c}$ can perfectly track any desired trajectory $\mathbf{u}_{c d}$ which has a piecewise continuous 3-rd derivative. This is true for all initial states $\mathbf{x}_{o}$ in $\mathcal{R}_{t i}+\mathcal{R}_{u c}$ and with piecewise continuous control functions $\mathbf{u}_{c}(t)$. Furthermore, it could be easily shown that the order 3 of the rigid-body object motions $\mathbf{u}_{c}$, is not due to the particular choice of the subspace $\mathcal{R}_{u c}$ but it is an inherent property of the system. It is related to the relative degree of the relationship between the rigid-body object motion and the joint-torques. Technical hypothesis $\mathbf{A 1}$ is introduced in order to simplify the theorem proof.

In (Prattichizzo and Bicchi, 1996) the output functional controllability was approached by proving the invertibility of the input-output representation of manipulation systems dynamics. Here the problem of the output functional controllability is approached by decoupling the force/motion control by means of a state-feedback controller. The improvement consists in obtaining a more robust controller in the sense that, if the inversion algorithm fails, the decoupling structure is able to fix at least the possible force/motion coupling problems. Furthermore, regarding computational aspects, the inversion of a block-diagonal transfer function usually involves less operations than the inversion of the undecoupled $\mathbf{G}(s)$.

\section{CASE STUDY}

Numerical results are reported for the simple defective gripper in Fig. 2. It is a planar 3-DoF's cartesian manipulator and has been chosen in order to show the effectiveness of previous results for industrial grippers.

In the base frame $\mathrm{B}$, the contact centroids, cf. (Bicchi et al., 1995), are $c_{1}=(2,2), \mathbf{c}_{2}=(2,3)$ and the object center of mass is $c_{b}=(2,2.5)$ while the transpose of the Jacobian and the grasp matrix assume the following values 


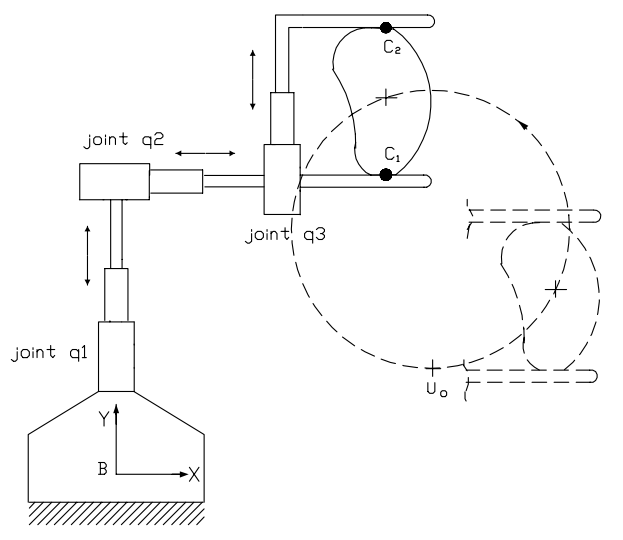

Fig. 2. Planar 3-DoF's cartesian manipulator. It exhibits a defective $\left(\operatorname{ker}\left(\mathbf{J}^{T}\right)=\{\mathbf{0}\}\right)$ grasp. System

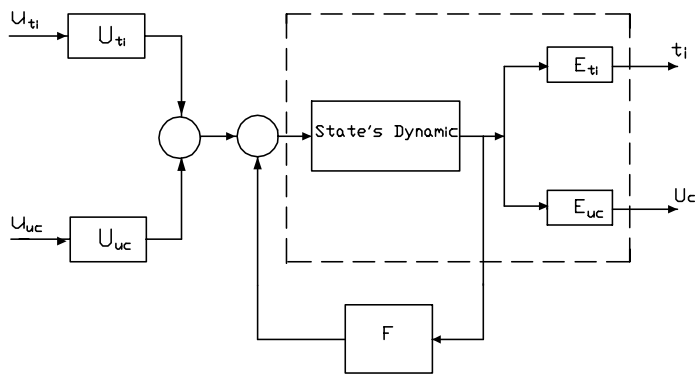

Fig. 3. Force/motion decoupling controller.

$$
\mathbf{J}^{T}=\left[\begin{array}{llll}
0 & 1 & 0 & 0 \\
1 & 0 & 1 & 0 \\
0 & 0 & 0 & 1
\end{array}\right] ; \quad \mathbf{G}=\left[\begin{array}{rrrr}
1 & 0 & 1 & 0 \\
0 & 1 & 0 & 1 \\
0.5 & 0 & -0.5 & 0
\end{array}\right]
$$

The inertia matrices of the object and manipulator along with stiffness and damping matrices at the contacts are assumed to be normalized to the identity matrix.

The controlled outputs are (a) the projection $\mathbf{t}_{i}$ of the contact forces along the 1-dimensional sub-

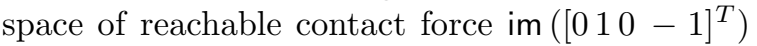
and (b) the projection of the rigid-body motion in the 2-dimensional subspace of object motions $\operatorname{im}\left[\begin{array}{ll}1 & 0 \\ 0 & 1 \\ 0 & 0\end{array}\right]$ which, since $\mathbf{u}=\left[\begin{array}{lll}\delta \mathbf{x} & \delta \mathbf{y} & \delta \theta\end{array}\right]^{T}$, corresponds to translations of the object.

The objective of the control is twofold. First, force and motion control must be decoupled, then the perfect tracking of desired trajectories $\mathbf{t}_{i d}$ and $\mathbf{u}_{c d}$ can be achieved.

The decoupling controller is pictorially described in Fig. 3 and has been synthesized, according to Section 3.1, eq. 5, 8 and 9. The state-feedback matrix $\mathbf{F}$ and the input partition matrices $\mathbf{U}_{t i}$ and $\mathbf{U}_{u c}$ are obtained respectively as

$$
\begin{gathered}
{\left[\begin{array}{cccccccccccc}
-7 & 6.5 & -6 & -1 & -41 & 0 & -7.5 & -0.02 & -5.5 & -3 & -22 & 0 \\
10 & -120 & 10 & -72 & 5 & 0 & 0.29 & -16 & 0.29 & 7.2 & -6.2 & 0 \\
-6.1 & 6.5 & -7.1 & -0.97 & -41 & 0 & -5.5 & -0.021 & -7.5 & -3.1 & -22 & 0
\end{array}\right]} \\
\\
{\left[\begin{array}{c}
-0.707 \\
0 \\
0.707
\end{array}\right] ; \quad\left[\begin{array}{cc}
0 & -0.707 \\
1 & 0 \\
0 & -0.707
\end{array}\right] .}
\end{gathered}
$$
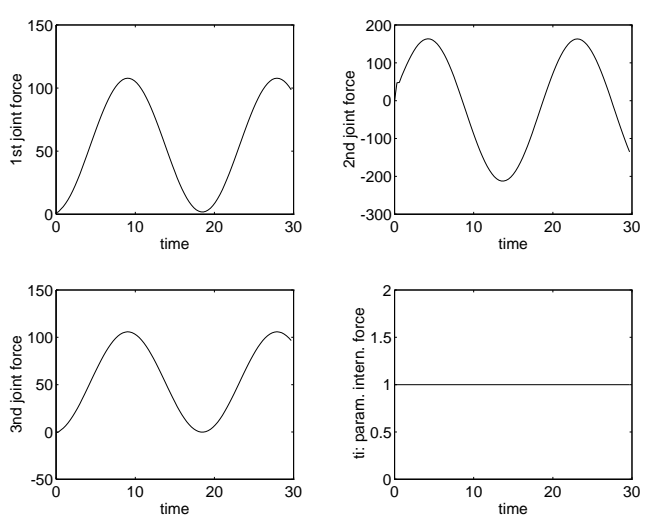

Fig. 4. Internal force $\mathbf{t}_{i}$ perfectly tracks the constant internal force while the object center of mass perfectly tracks the unit circle as depicted in Fig. 2.

The control task is set to follow a circle with angular velocity of $0.1 \mathrm{rad} / \mathrm{sec}$, starting from point $\mathbf{u}_{o}$ of coordinates $(2.5,1)$, see Fig. 2, while keeping the contact force constant to the value $\mathbf{t}_{o}=$ $[0 ; 1 ; 0 ;-1]^{T}$.

The computed prismatic joint forces $\tau^{*}=\mathbf{U}_{t i} \mathbf{u}_{t i}+$ $\mathbf{U}_{u c} \mathbf{u}_{u c}$ realizing the perfect tracking of desired object motions and internal force are reported in Fig. 4 along with the perfectly tracked internal force parameterization $\mathbf{t}_{i}$. The object trajectory perfect tracking is pictorially reported in Fig. 2 . For the choice of suitable inputs $\mathbf{u}_{t i}$ and $\mathbf{u}_{u c}$ the reader is referred to (Prattichizzo et al., 1997).

\section{CONCLUSIONS}

The decoupling and trajectory tracking procedure were discussed. The class of manipulation systems under investigation is wide enough to include a considerable number of grasp configurations, such as those using internal and/or extremal links to grasp objects. Due to the possible presence of defectivity, the control outputs were suitably chosen as the reachable internal forces and the rigid-body object motions.

The main results of the paper are summarized in two theorems. The first is related to noninteracting control. The second focuses on the perfect output functional controllability of internal force and object motions.

\section{REFERENCES}

Basile, G. and G. Marro (1971). On the perfect output controllability of linear dynamic systems. Ricerche di Automatica. 
Basile, G. and G. Marro (1992). Controlled and conditioned invariants in linear system theory. Prentice Hall. New Jersey.

Bicchi, A. and D. Prattichizzo (1995). A standard form for the dynamics of general manipulation systems. In: Proc. IEEE Int. Conf. Robotics and Automat.

Bicchi, A., C. Melchiorri and D. Balluchi (1995). On the mobility and manipulability of general multiple limb robots. IEEE Trans. on Automat. Contr.

Prattichizzo, D. and A. Bicchi (1996). Specifying consistent control goals for kinematically defective manipulation systems. In: Proceedings IEEE Internationl Conference on Robotics and Automation.

Prattichizzo, D. and A. Bicchi (1997). Dynamic analysis of mobility and graspability of general manipulation systems. IEEE Transactions on Robotics and Automation. To appear. Accepted 1997.

Prattichizzo, D., P. Mercorelli, A. Bicchi and A. Vicino (1996). Noninteracting force/motion control in general manipulation systems. In: Proceedings 35th IEEE International Conference on Decision Control.

Prattichizzo, D., P. Mercorelli, A. Bicchi and A. Vicino (1997). Geometric control techniques in controlling manipulation systems. Technical report.

Siciliano, B. (1996). Parallel force/position control of robotic manipulation. Springer-Verlag. London.

\section{Appendix:Proof of Theorem 2}

The proof of Theorem 2 is sketched in the present section. Further details on the proof can be found in (Prattichizzo et al., 1997).

According to (Basile and Marro, 1971), in order to prove Theorem 2, it will suffice to show that controlled invariants $\mathcal{R}_{t i}$ and $\mathcal{R}_{u c}$, used to synthesize the decoupling controller, satisfy the projection condition, reported below.

Lemma 1. (Projection condition) A generic (A, B)-controlled invariant $\mathcal{F}^{i}$ corresponds in the state space to the subspace $\mathcal{L}^{i}=\mathbf{E} \mathcal{F}^{i}$ of perfect output controllability with respect to the i-th derivative, see Definition 1, iff

$$
\mathcal{F}^{i} \subseteq \mathcal{F}^{i} \cap \mathcal{Z}_{i-1}+\operatorname{ker}(\mathbf{E})
$$

where $\mathcal{Z}_{i-1}$ is given by the recursion

$\mathcal{Z}_{0}=\operatorname{im}\left(\mathbf{B}_{\tau}\right)$

$\mathcal{Z}_{k}=\operatorname{im} \mathbf{B}_{\tau}+\mathbf{A}\left(\mathcal{Z}_{k-1} \cap \mathcal{F}^{i} \cap \operatorname{ker}(\mathbf{E})\right) \quad(k=1, \ldots, i-1)$.

\section{Proof of Theorem 2}

(a) Consider the constrained-controllability subspace of contact force, $\mathcal{R}_{t i}$ in eq. 10 . Being the projection condition fulfilled with $i=1$ :

$$
\begin{aligned}
& \mathcal{R}_{t i}^{1} \subseteq \operatorname{ker}\left(\mathbf{E}_{\mathrm{ti}}\right)+\mathcal{R}_{t i}^{1} \cap \operatorname{im}\left(\mathbf{B}_{\tau}\right) \quad \text { where } \\
& \operatorname{ker}\left(\mathbf{E}_{t i}\right)+\mathcal{R}_{t i}^{1} \cap \operatorname{im}\left(\mathbf{B}_{\tau}\right)= \\
& \operatorname{im}\left[\begin{array}{ccccccc|cc}
\boldsymbol{\Gamma}_{r} & \mathbf{0} & \boldsymbol{\Gamma}_{q c} & \mathbf{0} & \mathbf{0} & \mathbf{0} & \mathbf{I} & \mathbf{0} & \mathbf{0} \\
\mathbf{0} & \mathbf{0} & \mathbf{0} & \mathbf{0} & \mathbf{I} & \mathbf{0} & \mathbf{0} & \mathbf{0} & \mathbf{0} \\
\mathbf{0} & \boldsymbol{\Gamma}_{r} & \mathbf{0} & \boldsymbol{\Gamma}_{q c} & \mathbf{0} & \mathbf{0} & -\mathbf{I} & \Gamma_{h} & \mathbf{X} \\
\mathbf{0} & \mathbf{0} & \mathbf{0} & \mathbf{0} & \mathbf{0} & \mathbf{I} & \mathbf{0} & \mathbf{0} & \mathbf{0}
\end{array}\right],
\end{aligned}
$$

the column space $\operatorname{im}\left(\mathbf{E}_{t i}\right)=\mathbf{E}_{t i} \mathcal{R}_{t i}$ is of perfect output functionally controllable with respect to the 1st derivative and with respect to the subspace $\mathcal{R}_{t i}$, used for noninteraction.

(b) Consider the constrained--controllability subspace of object motions, $\mathcal{R}_{u c}$ in eq. 11 . Being the projection condition fulfilled with $i=3$ :

$$
\mathcal{R}_{u c}^{3} \subseteq \operatorname{ker}\left(\mathbf{E}_{\mathbf{u c}}\right)+\mathcal{R}_{u c}^{3} \cap \mathcal{Z}_{2},
$$

the column space $\operatorname{im}\left(\mathbf{E}_{u c}\right)=\mathbf{E}_{u c} \mathcal{R}_{u c}$ is of perfect output functionally controllable w.r.t. the $3 \mathrm{rd}$ derivative and w.r.t. the subspace $\mathcal{R}_{u c}$.

In the following some significant steps of the projection condition algorithm for the part (b) of the proof has been reported:

(1) $\mathcal{Z}_{0}=\operatorname{im}\left(\mathbf{B}_{\tau}\right)$

$\operatorname{ker}\left(\mathbf{E}_{u c}\right)+\mathcal{R}_{u c} \cap \operatorname{im}\left(\mathbf{B}_{\tau}\right)=\left[\begin{array}{cccc|c}\mathbf{I} & \mathbf{0} & \mathbf{0} & \mathbf{0} & \mathbf{0} \\ \mathbf{0} & \operatorname{ker}\left(\boldsymbol{\Gamma}_{u c}^{T}\right) & \mathbf{0} & \mathbf{0} & \mathbf{0} \\ \mathbf{0} & \mathbf{0} & \mathbf{I} & \mathbf{0} & \boldsymbol{\Gamma}_{q c} \\ \mathbf{0} & \mathbf{0} & \mathbf{0} & \mathbf{I} & \mathbf{0}\end{array}\right]$.

From eq. 2 and 12, it follows $\mathbf{D} \not \subset \operatorname{ker}\left(\Gamma_{u c}^{T}\right)$, thus

$$
\mathcal{R}_{u c} \nsubseteq \operatorname{ker}\left(\mathbf{E}_{\mathbf{u c}}\right)+\mathcal{R}_{u c}^{1} \cap \operatorname{im}\left(\mathbf{B}_{\tau}\right)
$$

(2) $\mathcal{Z}_{1}=\mathbf{B}_{\tau}+\mathbf{A}\left(\mathcal{Z}_{0} \cap \operatorname{im}\left(\mathcal{R}_{u c}^{3}\right) \cap \operatorname{ker}\left(\mathbf{E}_{u c}\right)\right)=$

$$
\left[\begin{array}{cc}
\mathbf{0} & \boldsymbol{\Gamma}_{q c} \\
\mathbf{0} & \mathbf{0} \\
\mathbf{M}_{h}^{-1} & \mathbf{0} \\
\mathbf{0} & \mathbf{M}_{o}^{-1} \mathbf{G B J}_{q c}
\end{array}\right]
$$

and being $\mathbf{D} \nsubseteq \operatorname{ker}\left(\Gamma_{u c}^{T}\right)$,

$$
\mathcal{R}_{u c} \not \operatorname{ker}\left(\mathbf{E}_{\mathbf{u c}}\right)+\mathcal{R}_{u c} \cap \mathcal{Z}_{1}
$$

(3) $\mathcal{Z}_{2}=\mathbf{B}_{\tau}+\mathbf{A}\left(\mathcal{Z}_{1} \cap \operatorname{im}\left(\mathcal{R}_{u c}^{3}\right) \cap \operatorname{ker}\left(\mathbf{E}_{u c}\right)\right)=$

$$
\left[\begin{array}{ccc}
\mathbf{0} & \boldsymbol{\Gamma}_{q c} & \mathbf{0} \\
\mathbf{0} & \mathbf{0} & \mathbf{M}_{o}^{-1} \mathbf{G K J} \boldsymbol{\Gamma}_{q c} \\
\mathbf{M}_{h}^{-1} & \mathbf{0} & \mathbf{0} \\
\mathbf{0} & \mathbf{M}_{o}^{-1} \mathbf{G B J} \boldsymbol{\Gamma}_{q c} & \mathbf{L}
\end{array}\right]
$$

where

$$
\mathbf{L}=\mathbf{M}_{o}^{-1} \mathbf{G K J} \Gamma_{q c}-\mathbf{M}_{o}^{-1} \mathbf{G B G}^{\mathbf{T}} \mathbf{M}_{o}^{-1} \mathbf{G B J} \Gamma_{q c} .
$$

From eq.2, it follows that

$$
\mathbf{M}_{o}^{-1} \mathbf{G K J} \boldsymbol{\Gamma}_{q c}=\mathbf{M}_{o}^{-1} \mathbf{G K G} \mathbf{T}^{T} \boldsymbol{\Gamma}_{u c}
$$


and, being $\operatorname{ker}\left(\mathbf{G}^{T}\right)=\{\mathbf{0}\}$, under assumption $\mathbf{A} \mathbf{1}$, it holds

$$
\operatorname{im}\left(\mathbf{M}_{o}^{-1} \mathbf{G} \mathbf{K J} \boldsymbol{\Gamma}_{q c}\right)+\operatorname{ker}\left(\boldsymbol{\Gamma}_{u c}^{T}\right)=\mathbb{R}^{d},
$$

where $d$ is the dimension of the vector $\mathbf{u}$ describing the object configuration.

Finally, by using the equation above, it is an easy matter to verify that the project condition is obtained for $i=3$ :

$$
\mathcal{R}_{u c}^{3} \subseteq \operatorname{ker}\left(\mathbf{E}_{\mathbf{u c}}\right)+\mathcal{R}_{u c}^{3} \cap \mathcal{Z}_{2} .
$$

